\title{
ON A THEOREM OF INGHAM ON NONHARMONIC FOURIER SERIES
}

\author{
ROBERT M. YOUNG
}

\begin{abstract}
A well-known result due to Ingham [3] shows that the system of complex exponentials $\left\{e^{i \lambda_{n} t}\right\}$ is a basic sequence in $L^{2}(-\pi, \pi)$ whenever $\lambda_{n+1}-\lambda_{n} \geq \gamma>1$. In this note, we show that the system need not be basic if $\lambda_{n+1}-\lambda_{n}>1$.
\end{abstract}

1. Introduction. Let $\left\{\lambda_{n}\right\}$ be an increasing sequence of real numbers. A wellknown result due to Ingham [3] states that if $\lambda_{n+1}-\lambda_{n} \geq \gamma>1$ then the series $\sum c_{n} e^{i \lambda_{n} t}$ converges in $L^{2}(-\pi, \pi)$ whenever $\sum\left|c_{n}\right|^{2}<\infty$ and, moreover,

$$
A \sum\left|c_{n}\right|^{2} \leq\left\|\sum c_{n} e^{i \lambda_{n} t}\right\|^{2} \leq B \sum\left|c_{n}\right|^{2} .
$$

Here, $A$ and $B$ are positive constants depending only on $\gamma$. (That the right-hand inequality is valid whenever $\gamma>0$ appears to have been first proved by Titchmarsh [10].)

Ingham showed that his result is the best possible in the sense that if $\gamma=1$ then the left-hand inequality cannot obtain. A counterexample is provided by the sequence $\left\{\lambda_{n}\right\}$ where

$$
\lambda_{n}= \begin{cases}n-1 / 4, & n>0, \\ n+1 / 4, & n<0 .\end{cases}
$$

It follows readily from (1) that the system of exponentials $\left\{e^{i \lambda_{n} t}\right\}$ is a basic sequence in $L^{2}(-\pi, \pi)$, that is, a basis for its closed linear span $S$. Accordingly, each function $f$ in $S$ has a unique representation

$$
f(t)=\sum c_{n} e^{i \lambda_{n} t} \quad \text { (in the mean). }
$$

The study of such nonharmonic Fourier series was initiated by Paley and Wiener [5] who showed that the system $\left\{e^{i \lambda_{n} t}\right\}$ is a basis for $L^{2}(-\pi, \pi)$ whenever the $\lambda_{n}$ are sufficiently close to the integers. Since then the theory has been generalized in many ways and in many different directions (see, e.g., $[\mathbf{2}, \mathbf{6}, \mathbf{8}, \mathbf{1 1}]$ and the references therein).

Condition (1), while tractable, is a stringent requirement to place on a basic sequence. Nevertheless, we show in Theorem 1 that the right-hand inequality must obtain for every basic sequence of exponentials. At present, there is no known example of such a sequence for which the left-hand inequality does not also obtain. Theorem 2 further dramatizes the strength of Ingham's result by showing that the slightly weaker separation condition $\lambda_{n+1}-\lambda_{n}>1$ cannot even guarantee that the system $\left\{e^{i \lambda_{n} t}\right\}$ is a basic sequence in $L^{2}(-\pi, \pi)$.

Received by the editors December 20, 1983.

1980 Mathematics Subject Classification. Primary 42C99; Secondary 46B15.

Key words and phrases. Nonharmonic Fourier series, basic sequence, biorthogonal system.

(C) 1984 American Mathematical Society $0002-9939 / 84 \$ 1.00+\$ .25$ per page 
THEOREM 1. If $\left\{\lambda_{n}\right\}$ is an increasing sequence of real numbers for which the system of exponentials $\left\{e^{i \lambda_{n} t}\right\}$ is a basic sequence in $L^{2}(-\pi, \pi)$, then the inequality $\left\|\sum c_{n} e^{i \lambda_{n} t}\right\|^{2} \leq B \sum\left|c_{n}\right|^{2}$ is valid for some constant $B$ and all square summable sequences of scalars $\left\{c_{n}\right\}$.

THEOREM 2. There exists a sequence $\left\{\mu_{n}\right\}$ of real numbers satisfying $\mu_{n+1}-$ $\mu_{n}>1$ such that $\left\{e^{i \mu_{n} t}\right\}$ is exact in $L^{2}(-\pi, \pi)$ and yet not a basis.

Recall that $\left\{e^{i \mu_{n} t}\right\}$ is said to be exact if it is complete but fails to be complete upon the removal of a single term.

2. Proof of Theorem 1. We need only show that the $\lambda_{n}$ are separated, i.e., that $\lambda_{n+1}-\lambda_{n} \geq \gamma$ for some positive constant $\gamma$; the result will then follow from [10].

Let $S$ be the closure in $L^{2}(-\pi, \pi)$ of the linear span of the system $\left\{e^{i \lambda_{n} t}\right\}$, and let $\left\{f_{n}\right\}, f_{n} \in S^{*}$, be the associated sequence of coefficient functionals. Then $\left\|e^{i \lambda_{n} t}\right\|\left\|f_{n}\right\| \leq M$ for some constant $M$ and all values of $n$ (see, e.g., $[\mathbf{9}$, p. 20]). Since each $\lambda_{n}$ is real, $\left\|e^{i \lambda_{n} t}\right\|=1$ and hence $\left\|f_{n}\right\| \leq M$. Now $f_{n}\left(e^{i \lambda_{n} t}-e^{i \lambda_{n+1} t}\right)=1$ so that $\left\|f_{n}\right\|\left\|e^{i \lambda_{n} t}-e^{i \lambda_{n+1} t}\right\| \geq 1$. Accordingly, $\left\|e^{i \lambda_{n} t}-e^{i \lambda_{n+1} t}\right\| \geq 1 / M$ and the existence of $\gamma$ follows.

3. Proof of Theorem 2. The system $\left\{e^{i \lambda_{n} t}\right\}$ where the $\lambda_{n}$ are given by (1), is known to be exact in $L^{2}(-\pi, \pi)[4, \mathrm{p} .67]$. We begin by showing that it is not a basis. Suppose it were. Then we could write

$$
1=\sum c_{n} e^{i \lambda_{n} t} \quad \text { (in the mean). }
$$

To compute the $c_{n}$, we shall make use of the Paley-Wiener space $P$ consisting of all entire functions of exponential type at most $\pi$ that are square integrable on the real axis. The inner product of two functions $F$ and $G$ in $P$ is, by definition,

$$
(F, G)=\int_{-\infty}^{\infty} F(x) \overline{G(x)} d x .
$$

By virtue of the Paley-Wiener theorem, the complex Fourier transform

$$
f(t) \rightarrow \frac{1}{2 \pi} \int_{-\pi}^{\pi} f(t) e^{i z t} d t
$$

is an isometric isomorphism from $L^{2}(-\pi, \pi)$ onto all of $P$. The exponentials $e^{i \lambda_{n} t}$ are sent to the "reproducing" functions

$$
K_{n}(z)=\frac{\sin \pi\left(z-\lambda_{n}\right)}{\pi\left(z-\lambda_{n}\right)}
$$

which then consistute a basis for $P$. Let $\left\{g_{n}\right\}$ be biorthogonal to $\left\{K_{n}\right\}$. When the Fourier transform is applied to (3), we obtain

$$
\frac{\sin \pi z}{\pi z}=\sum c_{n} K_{n}(z)
$$

where $c_{n}=\left((\sin \pi z) / \pi z, g_{n}\right)=g_{n}(0)$.

Let

$$
F(z)=\prod_{n=1}^{\infty}\left(1-\frac{z^{2}}{\lambda_{n}^{2}}\right) .
$$


It was shown by Levinson $[4$, p. 67] that

$$
F(z)=c \int_{-\pi}^{\pi}\left(\cos ^{-1 / 2} \frac{1}{2} t\right) e^{i z t} d t .
$$

Since $\cos ^{-1 / 2} \frac{1}{2} t$ is integrable over $(-\pi, \pi)$, it follows that $F(z)$ is bounded along the real axis and each of the functions

$$
F_{n}(z)=F(z) / F^{\prime}\left(\lambda_{n}\right)\left(z-\lambda_{n}\right)
$$

therefore belongs to $P$. Since $\left(F_{n}, K_{m}\right)=F_{n}\left(\lambda_{m}\right)=\delta_{m n}$, it follows that $F_{n}=g_{n}$. Thus

$$
c_{n}=F_{n}(0)=-1 / \lambda_{n} F^{\prime}\left(\lambda_{n}\right)
$$

and (3) becomes

$$
1=-\sum_{n \neq 0} \frac{e^{i \lambda_{n} t}}{\lambda_{n} F^{\prime}\left(\lambda_{n}\right)}=-2 \sum_{n=1}^{\infty} \frac{\cos \lambda_{n} t}{\lambda_{n} F^{\prime}\left(\lambda_{n}\right)}
$$

since $z F^{\prime}(z)$ is even. It is to be shown that the series on the right does not converge in $L^{2}(-\pi, \pi)$.

Now the values of $F^{\prime}\left(\lambda_{n}\right)$ were determined explicitly in [7]:

$$
F^{\prime}\left(\lambda_{n}\right)=(-1)^{n} \Gamma^{2}\left(\frac{3}{4}\right) \frac{\Gamma(n)}{\Gamma\left(n+\frac{1}{2}\right)} \quad(n=1,2,3, \ldots) .
$$

Using the asymptotic formula $\Gamma(n) / \Gamma\left(n+\frac{1}{2}\right)=1 / \sqrt{n}+O\left(1 / n^{3 / 2}\right)[\mathbf{1}]$, we have

$$
F^{\prime}\left(\lambda_{n}\right)=A(-1)^{n}\left\{1 / \sqrt{\lambda_{n}}+\varepsilon_{n}\right\} \quad \text { where } \varepsilon_{n}=O\left(1 / n^{3 / 2}\right) .
$$

A straightforward calculation then shows that the difference between the series on the right in (4) and

$$
\frac{1}{A} \sum_{n=1}^{\infty} \frac{(-1)^{n} \cos \lambda_{n} t}{\sqrt{\lambda_{n}}}
$$

is uniformly convergent on $[-\pi, \pi]$. Accordingly, we need only show that this series diverges in $L^{2}(-\pi, \pi)$.

Let $x=\pi-t(0 \leq t \leq \pi)$. Then

$$
\begin{aligned}
\sum_{n=1}^{\infty} \frac{(-1)^{n}}{\sqrt{\lambda_{n}}} & =\sum_{n=1}^{\infty} \frac{(-1)^{n} \cos \left(\pi n-\pi / 4-\lambda_{n} x\right)}{\sqrt{\lambda_{n}}} \\
& =\sum_{n=1}^{\infty} \frac{\cos \left(\pi / 4+\lambda_{n} x\right)}{\sqrt{\lambda_{n}}}
\end{aligned}
$$

For $N=1,2,3, \ldots$, let $\delta_{N}=\pi / 16 N$. If $x \in\left[0, \delta_{N}\right]$, then $\pi / 4+\lambda_{n} x \in[\pi / 4,3 \pi / 8]$ whenever $1 \leq n \leq 2 N$ and hence $\cos \left(\pi / 4+\lambda_{n} x\right) \geq A>0$. Thus

$$
\sum_{N}^{2 N} \frac{\cos \left(\pi / 4+\lambda_{n} x\right)}{\sqrt{\lambda_{n}}} \geq A \sum_{N}^{2 N} \frac{1}{\sqrt{n-1 / 4}} \geq A \frac{N+1}{\sqrt{2 N-1 / 4}} \geq B \sqrt{N}
$$


where $B$ is a positive constant independent of $N$. Accordingly,

$$
\begin{aligned}
\left\|\sum_{N}^{2 N} \frac{(-1)^{n} \cos \lambda_{n} t}{\sqrt{\lambda_{n}}}\right\|^{2} & \geq\left\|\sum_{N}^{2 N} \frac{\cos \left(\pi / 4+\lambda_{n} x\right)}{\sqrt{\lambda_{n}}}\right\|_{L^{2}\left(0, \delta_{N}\right)}^{2} \\
& \geq \frac{1}{2 \pi} B^{2} N \delta_{N}=\frac{B^{2}}{32}
\end{aligned}
$$

for all $N$. Thus the series in (3) does not converge in $L^{2}(-\pi, \pi)$, and the system $\left\{e^{i \lambda_{n} t}\right\}$ fails to be a basis.

Let $\left\{\varepsilon_{1}, \varepsilon_{2}, \ldots\right\}$ be a decreasing sequence of positive numbers such that $\varepsilon_{1}<\frac{1}{4}$ and $\sum \varepsilon_{n}<\infty$. It is to be shown that if $\mu_{n}=\lambda_{n}-\varepsilon_{n}, \mu_{-n}=-\mu_{n}(n=1,2, \ldots)$, then the system $\left\{e^{i \mu_{n} t}\right\}$ satisfies the conclusions of the theorem.

Clearly, $\mu_{n+1}-\mu_{n}>1$. Since $\sum\left|\lambda_{n}-\mu_{n}\right|<\infty$ and $\left\{e^{i \lambda_{n} t}\right\}$ is exact, so is $\left\{e^{i \mu_{n} t}\right\}[6]$. It remains only to show that $\left\{e^{i \mu_{n} t}\right\}$ is not a basis for $L^{2}(-\pi, \pi)$. Suppose it were. Then the system $\left\{h_{n}(t)\right\}$, biorthogonal to $\left\{e^{i \mu_{n} t}\right\}$, would satisfy $\left\|e^{i \mu_{n} t}\right\| \cdot\left\|h_{n}\right\| \leq M$ for some constant $M$ and all values of $n$. Since each $\mu_{n}$ is real, $\left\|e^{i \mu_{n} t}\right\|=1$ and hence $\left\|h_{n}\right\| \leq M$. We complete the proof by showing that

$$
\sum\left|\lambda_{n}-\mu_{n}\right|<\infty \Rightarrow \sum\left\|e^{i \lambda_{n} t}-e^{i \mu_{n} t}\right\|<\infty .
$$

The convergence of $\sum\left\|e^{i \lambda_{n} t}-e^{i \mu_{n} t}\right\|\left\|h_{n}\right\|$ will then imply that $\left\{e^{i \lambda_{n} t}\right\}$ is a basis for $L^{2}(-\pi, \pi)$ (see, e.g., $[\mathbf{9}$, p. 94]). The contradiction will prove the theorem.

To establish (5), write

$$
e^{i \lambda_{n} t}-e^{i \mu_{n} t}=e^{i \lambda_{n} t}\left(1-e^{-i \varepsilon_{n} t}\right)
$$

Expanding $1-e^{i \gamma t}$ in an everywhere-convergent Taylor series, we find

$$
\left|e^{i \lambda_{n} t}-e^{i \mu_{n} t}\right| \leq \sum_{K=1}^{\infty} \frac{\varepsilon_{n}^{K} t^{K}}{K !},
$$

and hence

$$
\begin{aligned}
\sum_{n}\left\|e^{i \lambda_{n} t}-e^{i \mu_{n} t}\right\| & \leq \sum_{K=1}^{\infty} \frac{\pi^{K}}{K !}\left(\sum_{n} \varepsilon_{n}^{K}\right) \leq \sum_{K=1}^{\infty} \frac{\pi^{K}}{K !}\left(\sum \varepsilon_{n}\right)^{K} \\
& =\exp \left(\pi \sum \varepsilon_{n}\right)-1<\infty
\end{aligned}
$$

This completes the proof.

\section{REFERENCES}

1. E. T. Copson, Asymptotic expansions, Cambridge Univ. Press, 1965.

2. S. V. Hruscev, N. K. Nikolskii and B. S. Pavlov, Unconditional bases of exponentials and of reproducing kernels, Lecture Notes in Math., vol. 864, Springer-Verlag, 1981, pp. 214-335.

3. A. E. Ingham, Some trigonometrical inequalities with applications to the theory of series, Math. Z. 41 (1936), 367-379.

4. N. Levinson, Gap and density theorems, Amer. Math. Soc. Colloq. Publ., vol. 26, Amer. Math. Soc., Providence, R.I., 1940

5. R. E. A. C. Paley and N. Wiener, Fourier transforms in the complex domain, Amer. Math. Soc. Colloq. Publ., vol. 19, Amer. Math. Soc., Providence, R.I., 1934.

6. R. M. Redheffer, Completeness of sets of complex exponentials, Adv. in Math. 24 (1977), 1-62. 
7. R. M. Redheffer and R. M. Young, Completeness and basis properties of complex exponentials, Trans. Amer. Math. Soc. 277 (1983), 93-111.

8. L. Schwartz, Etude des sommes d'expontielles, Publ. Inst. Math. Univ. Strasbourg, 2nd ed., vol. V, Hermann, Paris, 1959.

9. I. Singer, Bases in Banach spaces. I, Springer-Verlag, Berlin and New York, 1970.

10. E. C. Titchmarsh, A class of trigonometrical series, J. London Math. Soc. 3 (1928), 300-304.

11. R. M. Young, An introduction to nonharmonic Fourier series, Academic Press, New York, 1980.

Department of Mathematics, Oberlin College, Oberlin, Ohio 44074 